\title{
Controlling the Charge Carrier Density of Black Phosphorus in a Rapid Plasma Doping Process
}

\author{
Received July 17, 2020; revised November 6, 2020; accepted November 10, 2020
}

Dae-Kyoung Kim, Seok-Bo Hong, and Mann-Ho Cho*
Department of Physics, Yonsei University, Seoul O3722, Republic of Korea

*Corresponding author E-mail: mh.cho@yonsei.ac.kr

\begin{abstract}
We investigated the control of the charge carrier density of black phosphorus (BP) 2D nanosheets in a rapid plasma doping process. The electronic structure of plasma boron-doped BP was investigated using X-ray photoelectron spectroscopy and ultraviolet photoelectron spectroscopy. The characteristics of a doped BP device were evaluated by fabricating a field-effect transistor under spatially controlled doping in BP nanosheets. The results confirmed the incorporation of ionized boron through the plasma doping process, resulting in the donation of strongly electron dope (electron mobility: $\sim 275 \mathrm{~cm}^{2} / \mathrm{V} \cdot \mathrm{s}$ ) on the boron-doped BP switching device. In particular, the fabricated p-n homojunction of the BP device showed an excellent photodetection behavior. This work provides a rapid and stable plasma doping technique for two-dimensional nanosheets employed in next-generation electronic and optoelectronic devices.
\end{abstract}

Keywords: Black phosphorus, Plasma doping, Homojunction, Optoelectronic device

\section{Introduction}

Two-dimensional (2D) layered materials with a heterojunction have attracted attention in recent years owing to their unique physical properties (high mobility and ultimate flexibility) and promising applications in new-generation electronic and optoelectronic devices [1-3]. Among the many 2D applications, photovoltaic-based 2D layered material devices exhibit both $\mathrm{n}$ - or $\mathrm{p}$-type unipolar characteristics in semiconductors composed of the same material (i.e., homojunction). These homojunctions of the 2D materials can be formed stably and can help reduce the number of defect states in the interface region owing to their perfectly ordered microstructure. Thus, many $2 \mathrm{D}$ materials along with the modulation of the carrier-type method have been actively researched. However, controlling the carrier type in 2D materials for work function engineering or separated charge conversion remains difficult, and an unstable distribution is sometimes formed. Among 2D materials with unique physical properties, black phosphorus (BP) has shown a band gap ranging from $0.3 \mathrm{eV}$ (for its bulk crystal) to $2.0 \mathrm{eV}$ (for the monolayer) as the thickness is reduced up to the monolayer under direct energy band-gap [4-6]. The notable thicknessmodulated energy gap and its characteristics have enabled the application of such materials to optoelectronic devices. Moreover, BP can exhibit a hole-dominated ambipolar carrier for a high hole mobility $\left(\sim 1000 \mathrm{~cm}^{2} / \mathrm{V} \cdot \mathrm{s}\right)$ at room temperature. However, the deviation in the characteristics of the carrier type has made it difficult to create complementary logic devices on a single BP semiconductor. Thus, controlling the charge carrier density is important to tune the n- or p-type energy level of the BP system and thereby improve the performance of BP-based electronic and optoelectronic devices.

In this work, we evaluated the control of the charge carrier density of 2D BP nanosheets in a rapid plasma boron doping process. The results confirm boron incorporation through the pulsed-plasma doping process, resulting in the donation of surface electron dope on BP. Furthermore, the fabricated $\mathrm{p}^{-} \mathrm{n}$ homojunction of the BP photovoltaic device showed an excellent photodetection behavior.

\section{Experimental details}

Exfoliated BP was prepared using the standard scotch tape method and transferred onto a $300 \mathrm{~nm} \mathrm{SiO} / / \mathrm{Si}$ think film containing $\mathrm{Au}$ electrode markers. The height and optical image contrast of the BP nanosheets were confirmed by atomic force microscopy, and metal $(\mathrm{Ti} / \mathrm{Au})$ electrodes were patterned by e-beam lithography. After exposing the BP flakes to the plasma doping process, we preserved the samples in a vacuum storage. The plasma-doped samples were investigated under vacuum conditions (X-ray photoelectron spectroscopy (XPS) and ultraviolet photoelectron spectroscopy (UPS) measurements). The plasma doping process was performed using $\mathrm{BF}_{3}$ (a dopant gas). As optimal process parameters, the plasma power, accelerating voltage, and working pressure were set to $100 \mathrm{~W}, 4000 \mathrm{~V}$, and $1 \times 10^{-2}$ Torr, respectively. The properties of the chemical bonding states of the plasma-processed BP were investigated using high-resolution XPS with a monochromatic $\mathrm{Al} K \alpha \mathrm{X}$-ray source $(h v=1486.7 \mathrm{eV})$ having a pass energy of $20 \mathrm{eV}$. The spectrum of each orbital characteristic was calibrated using the binding energy of carbon $(284.5 \mathrm{eV})$. To deconvolute the XPS core-level spectra, the background was eliminated using the Shirley-type subtraction, and the full width at half maximum values of the constituent peaks were kept constant. The fitting curves followed Gaussian and Lorentzian distributions. In terms of the photo-switching properties, the laser diode $(785 \mathrm{~nm})$ was operated in 
the wave mode. A laser beam was passed through an optical lens and was subsequently incident onto the device channel without overlap focusing (channel area: $<10 \mu \mathrm{m} \times 10 \mu \mathrm{m}$ ). The I-V properties of the BP device were investigated using two Keithley 2400 Source Meters at room temperature. The device mobility was calculated using the following equation,

$$
\mu_{e f f}=L g_{d} / W C_{o x} V_{s d}
$$

where $\mu_{\text {eff }}$ is the field-effect mobility, $L$ is the length of the channel region, $g_{d}$ is the transconductance, $W$ is the width of the channel region, $C_{\mathrm{ox}}$ is the capacitance of the oxide, and $V_{\text {sd }}$ is the source-drain voltage [7].

\section{Results and discussion}

First, we evaluated the changes in the surface chemical bonds on the BP surface with the boron interaction process. Figure 1(a) presents the chemical bonding states of the $1 s$ orbital of boron after the plasma process (pulsed process $(30 \mathrm{~s})$ ) with increasing pulse time (interval: 5 s) on the BP surface. The properties of the chemical bonding states for the relationship of boron atoms with BP (O-B-P, B-B, and B-P bonding states) were defined at a detection angle of $45^{\circ}[8,9]$. To fit the experimental data to a theoretical function, the Shirley-type background subtraction was applied to the boron spectra, and the charge was compensated by C-C bonds $(284.5 \mathrm{eV})$. The fitting curves show the existence of hybridized B-P states after employing the pulsed plasma process on the BP surface. Moreover, we found small hybridized O-B-P oxidized states resulting from the BP surface oxidation during the plasma process. The change in the plasma doping effect can be confirmed from the P $2 p$ orbital spectra, as shown in Fig. 1(b). The orbital spectrum of non-doped BP (pristine $\mathrm{BP}$ ) shows a characteristic doublet representing P $2 p_{3 / 2}(130.23 \mathrm{eV})$ and $2 p_{1 / 2}(131.08 \mathrm{eV})[10,11]$. In particular, after the boron plasma doping of $\mathrm{BP}$, the $\mathrm{P} 2 p_{3 / 2}$ peak shifts to the higher binding energy, from 130.23 to $130.34 \mathrm{eV}$ (an increase of $0.11 \mathrm{eV}$ ). This shift in the binding energy shows evidence for band bending, which indicates a vacuum-level shift with the downward band bending in the BP-layered material. To confirm the detailed band bending of the plasma-doped BP sample, a UPS measurement was performed. Figure 2 shows the difference between the work function value [Fig. 2(a)] and calibrated $\mathrm{E}_{\mathrm{F}}$ [Fig. 2(b)] of the boron plasma-doped BP and pristine BP. The work function of the plasma-doped BP decreases by $\triangle \mathrm{E}=0.2 \mathrm{eV}$, which contributes to the electron charge carrier doping and the formation of an interface dipole in the boron-doped BP surface region during the plasma doping process. Figures $3(\mathrm{a})$ and $3(\mathrm{~b})$ show a comparison of the
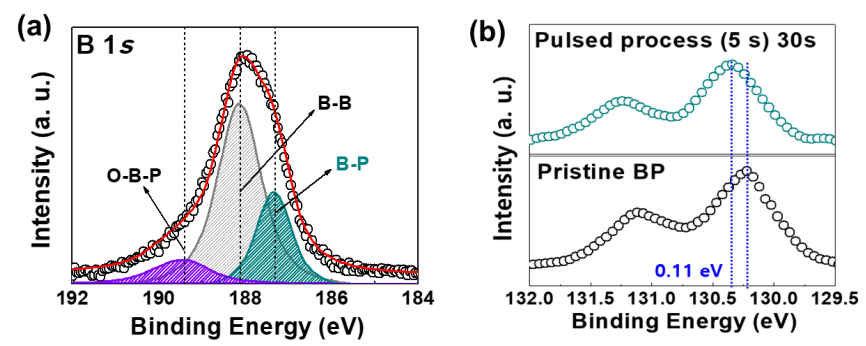

Figure 1. (a) B $1 s$ and (b) P 2p XPS spectra of pristine and plasma boron-doped BP. carrier transports of pristine BP and plasma-doped BP employed in field-effect transistor (FET) devices (Ti/Au metal electrode and BP $(\sim 13.7 \mathrm{~nm}))$. The transfer I-V curve indicates a p-type behavior with a mobility of $\sim 169 \mathrm{~cm}^{2} / \mathrm{V} \cdot \mathrm{s}$ in the BP pristine sample and an n-type conductance behavior with a mobility of $\sim 275 \mathrm{~cm}^{2} / \mathrm{V} \cdot \mathrm{s}$ in the plasmadoped BP at room temperature ( $295 \mathrm{~K})$, accompanied by an increase in the current in the hole and electron regimes. Subsequently, to evaluate the device characteristics of the $p^{-n}$ homojunction, a $p^{-n}$ junction device was fabricated by doping spatially controlled plasma on the BP flake sample ( $\mathrm{p}$ and $\mathrm{n}$ regions, $\sim 10 \mu \mathrm{m}$ ), as shown in Fig. $3(c)$. The $I_{d}-V_{d}$ curve indicates a rectification behavior in the $p-n$ junction device at gate voltages ranging from 4 to $-4 \mathrm{~V}$ with a step of $2 \mathrm{~V}$ [Fig. 3(d)], and the $\mathrm{I}_{\mathrm{d}}-\mathrm{V}_{\mathrm{d}}$ curves plotted on a logarithmic scale indicate a diode-like current rectifying characteristic [Fig. 3(e)]. This current rectification can be controlled using the gate voltage under forward and reverse mode voltages $\left(\mathrm{V}_{\mathrm{d}}\right)$. Consequently, a high rectification ratio of $2.8 \times 10^{4}$ was found at $V_{d}=-2 /+2 \mathrm{~V}$ and $V_{g}=-4$ $\mathrm{V}$, as shown in Fig. 3(f). Finally, to confirm the photovoltaic efficiency of the boron plasma-doped BP $\mathrm{p}^{-} \mathrm{n}$ homojunction, the photoinduced characteristics were evaluated. Figure 4(a) shows the time-dependent photoresponse at an incident laser power ranging from 100 to 400 $\mu \mathrm{W}$. The photoresponse on/off switching signal was well retained. Furthermore, the temporal responses of the rise and decay times were found to be $\sim 1.2$ and $7.6 \mathrm{~ms}$, respectively, as shown in Figs. 4(c) and $4(\mathrm{~d})$. The responsivity $(R)$ was calculated using the following equation,

$$
R=I_{\text {ph }} / P_{\text {in }}
$$

where the photocurrent $I_{p h}=I_{o n}-I_{o f f}$, and $P_{\text {in }}$ is the input power for the plasma-doped BP $\mathrm{p}^{-} \mathrm{n}$ homojunction. The maximum photoresponsivity was $11.3 \mathrm{~mA} / \mathrm{W}$, and the characteristics of $R$ increased with the decrease in the incident laser power. The combination of high electron mobility, on-off photoresponse time, and high responsivity at a low source-drain voltage $\left(\mathrm{V}_{\mathrm{d}}=0.1 \mathrm{~V}\right)$ suggests that the $\mathrm{p}-\mathrm{n}$ homojunction of the boron-doped BP device is sensitive to the separation efficiency of the photoexcited electron-hole pairs and shows promise for applications in electronic and optoelectronic devices. (a)

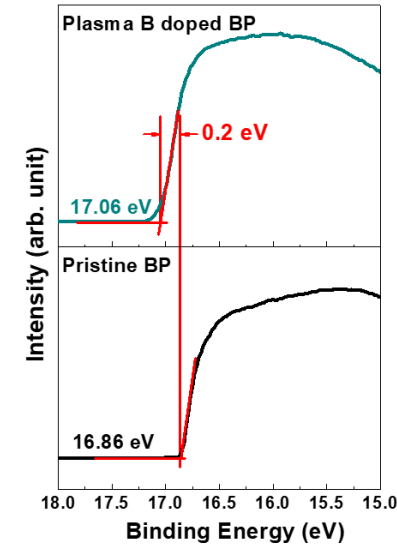

(b)

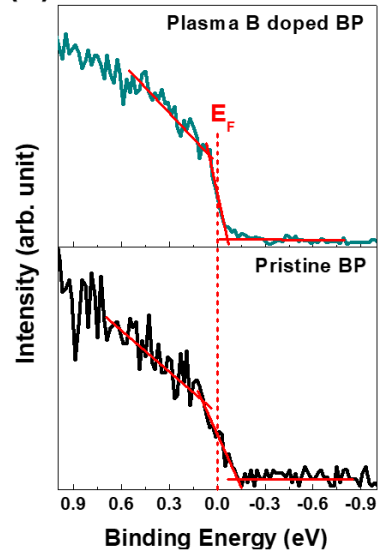

Figure 2. UPS of pristine BP and plasma boron-doped BP. The lines show the deviations in the secondary electron cutoff. 

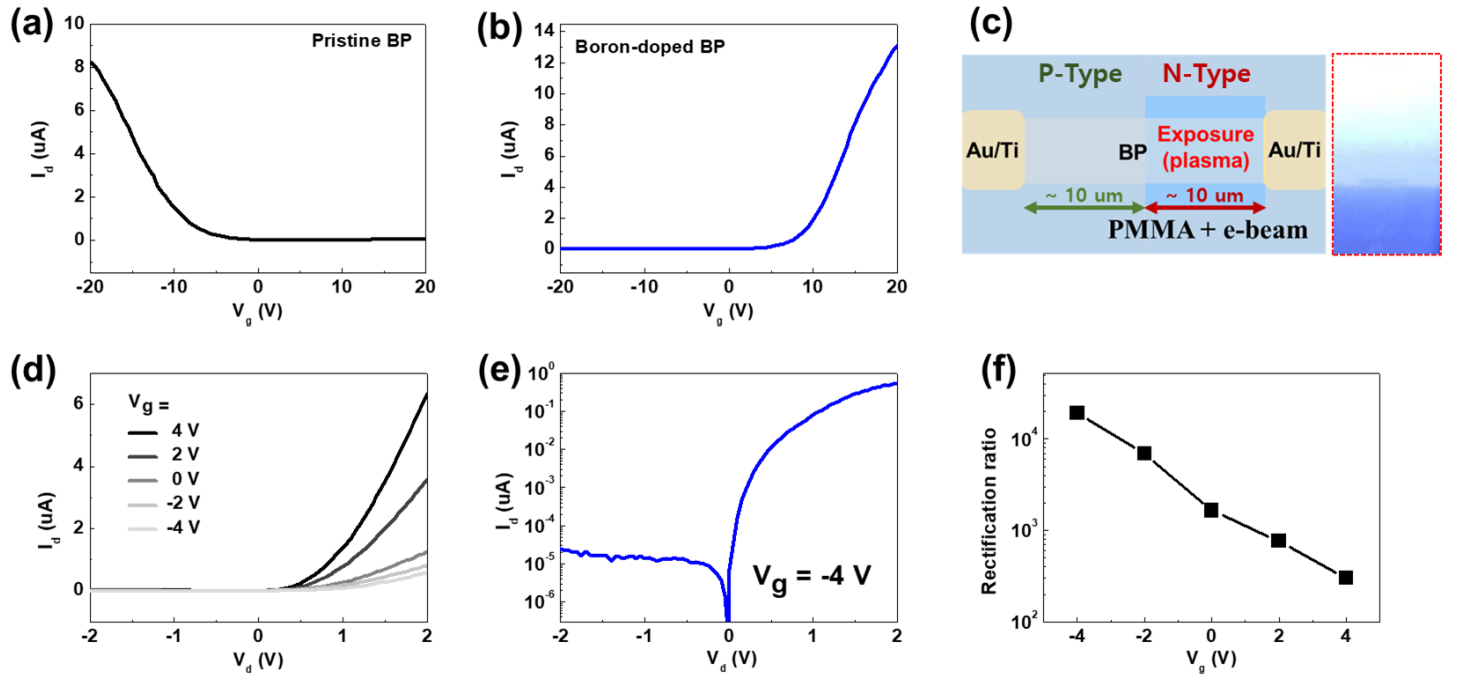

Figure 3. Comparison of $I_{d}-V_{g}$ transfer curves of (a) pristine and (b) plasma boron-doped BP at a source-drain voltage of $100 \mathrm{mV}$. (c) Scheme of BP $p-n$ homojunction. (d) Gate-tunable $I_{d}-V_{d}$ output curve of the boron-doped BP $p-n$ homojunction, and its (e) logarithmic scale at $-4 \mathrm{~V}$ and (f) rectification ratio.
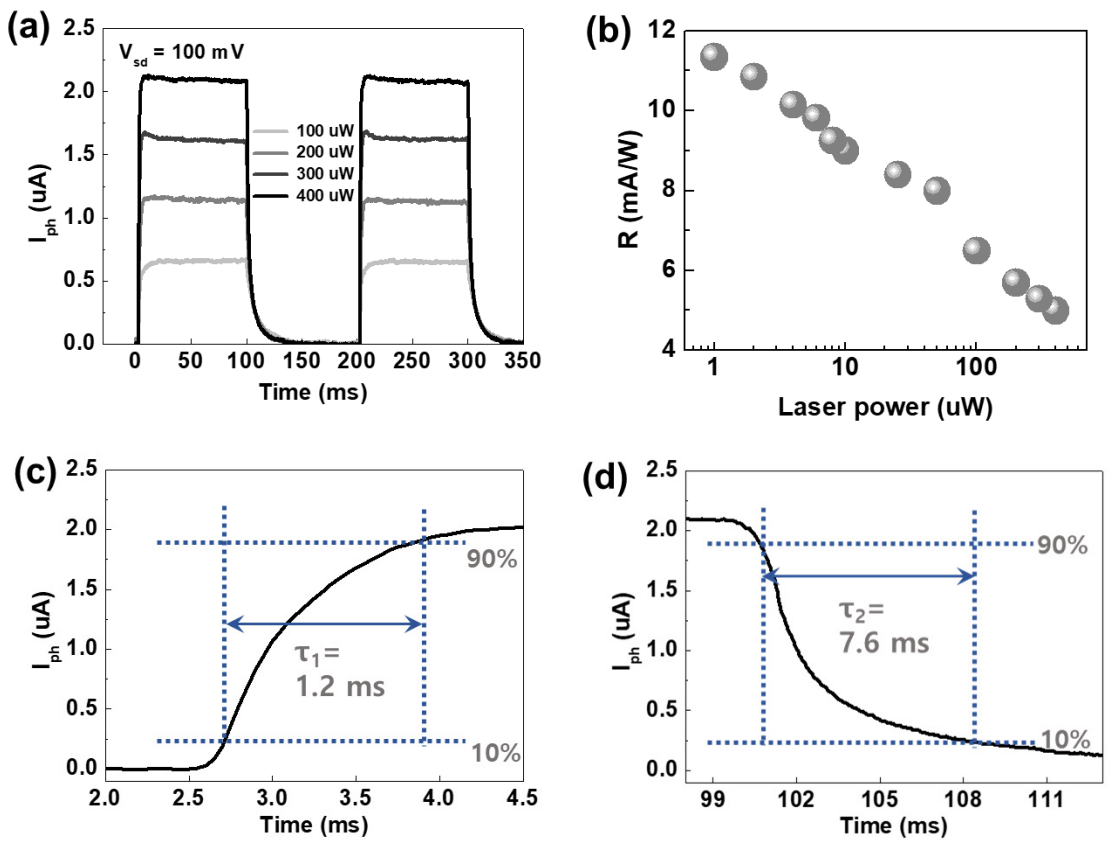

Figure 4. (a) Photoresponse switching at incident powers ranging from 100 to $400 \mu \mathrm{W}$. (b) Photoresponsivity of the plasma boron-doped BP $p-n$ homojunction. The temporal responses of the (c) rise and (d) decay times of the BP $p-n$ homojunction.

\section{Conclusions}

In summary, we investigated the control of the charge carrier density of black phosphorus (BP) 2D nanosheets in a rapid plasma boron doping process. We confirmed boron incorporation following the plasma boron doping process, resulting in the donation of strongly electron dope on BP. In particular, the plasma boron-doped BP $\mathrm{p}-\mathrm{n}$ homojunction device showed an excellent rectification and photodetection behavior. This work provides a rapid and stable plasma doping technique for $2 \mathrm{D}$ nanosheets employed in next-generation electronic and optoelectronic devices.

\section{Acknowledgements}

This research was supported by the National Research Foundation of Korea (NRF) grant funded by the Korea government (Grant No. 2017R1A5A1014862, SRC program vdWMRC center).

\section{References}

[1] K. Dolui, I. Rungger, and S. Sanvito, Phys. Rev. B 87, 165402 (2013).

[2] R. Cheng, D. Li, H. Zhou, C. Wang, A. Yin, S. Jiang, Y. Liu, Y. Chen, Y. Huang, and X. Duan, Nano Lett. 14, 5590 (2014). 
[3] Y. Li, J. K. Qin, C. Y. Xu, J. Cao, Z. Y. Sun, L. P. Ma, P. A. Hu, W. Ren, and L. Zhen, Adv. Funct. Mater. 26, 4319 (2016).

[4] Y. Du, C. Ouyang, S. Shi, and M. Lei, J. Appl. Phys. 107, 1 (2010).

[5] Ø. Prytz and E. Flage-Larsen, J. Phys. Condens. Matter 22 (2010).

[6] F. Ahmed, Y. D. Kim, M. S. Choi, X. Liu, D. Qu, Z. Yang, J. Hu, I. P. Herman, J. Hone, and W. J. Yoo, Adv. Funct. Mater. 27, 1 (2017).

[7] D. K. Schroder, Semiconductor Material and Device Characterization (IEEE Press, Wiley, 2006).
[8] B. Feng, J. Zhang, Q. Zhong, W. Li, S. Li, H. Li, P. Cheng, S. Meng, L. Chen, and K. Wu, Nat. Chem. 8, 563 (2016).

[9] G. Effenberg and S. Ilyenko, Refractory Metal Systems (Springer 2010).

[10] W. Luo, D. Y. Zemlyanov, C. A. Milligan, Y. Du, L. Yang, Y. Wu, and P. D. Ye, Nanotechnol. 27, 434002 (2016).

[11] D. K. Kim, J. Chae, S. B. Hong, H. Park, K. S. Jeong, H. W. Park, S. R. Kwon, K. B. Chung, and M. H. Cho, Nanoscale 10, 22896 (2018). 